\title{
Proceedings of the meeting of the Association of British Neurologists held jointly with the Society of British -Neurological Surgeons, in Edinburgh, 20-22 September
} 1984

$>$

AUTOSOMAL DOMINANT TYPE I HEREDITARY - MOTOR AND SENSORY NEUROPATHY (HMSN) NOT LINKED TO DUFFY. REPORT OF AN ENGLISH FAMILY

RJ Guiloff, PK Thomas, Marcela Contreras. Departments of Neurology, Westminster Hospital, Royal Free Hospital School of Medicine and North London Blood Transfusion Centre

Five individuals belonging to three generations of an English family with two members affected by HMSN type I were studied. HMSN I was shown to segregate independently from Duffy (Fy). These findings and those of two similar families reported in the literature (Bird et al 1983, Dyck et al 1983) support the idea of genetic heterogeneity within autosomal dominant type I HMSN.

\section{EARLY RECOVERY AFTER STROKE}

DT Wade, R Langton Hewer. Department of Neurology, Frenchay Hospital, Bristol

Physicians are frequently asked to give a prognosis for recovery in patients who suffered a stroke a few days previously. The majority of published data relate to recovery in patients who have been first assessed at three or four weeks post-stroke, and there is little information relating to recovery in the first few days.

This study was based on 99 patients who had various abilities recorded regularly over the first 13 weeks. Thirty two patients died before 13 weeks. Forty five of the 67 survivors had assessments twice weekly from within four days of their stroke. Five functional areas were studied; urinary continence, mobility, feeding, the ability to dress and the ability to transfer from bed to chair.

Recovery in the 45 patients occurred fastest in the first two weeks, by which time at least $50 \%$ of recovery had occurred, but was still continuing at 13 weeks. Over $50 \%$ of those initially dependent in any one activity regained independence by three months.
The most important adverse prognostic factor of those measured, both for survival and for recovery of function, was urinary incontinence. For instance, $100 \%$ of patients who were continent by $7-10$ days regained the ability to walk. Only $30 \%$ of the incontinent group did so. Age was the second most important factor.

DOES HAEMATOCRIT PREDICT DEATH AND FUNCTIONAL OUTCOME AFTER STROKE: THE EXPERIENCE OF THE OXFORDSHIRE

COMMUNITY STROKE PROJECT

JM Bamford, PAG Sandercock, CP Warlow. University Department of Clinical Neurology, Oxford

Hospital based series suggest that raised haematocrit is associated with increased mortality after stroke. We examined the relationship between haematocrit and both morbidity and mortality in $\mathbf{3 2 3}$ consecutive cases of first stroke from our community based register. Blood urea and haematocrit were measured as soon after the stroke as possible. Ninety per cent of patients had a CT scan and/or post mortem examination.

Life table analysis of the whole sample showed that increasing age, impaired consciousness at onset and cerebral haemorrhage were associated with increased 30 day mortality. There was no clear relationship between haematocrit and mortality.

We assessed the relationship between mortality, morbidity, haematocrit and type of cerebral infarction. Thirty day mortality was significantly higher in patients with supratentorial large vessel occlusion than with lacunar infarction, but there was no relationship between raised haematocrit and mortality.

Of survivors who had minimal or no stroke-related disability at one month, $49 \%$ with lacunar infarction and $34 \%$ with supratentorial large vessel occlusion had had an initial haematocrit $>0.45$. In those with moderate to severe disability, the proportions were $61 \%$ and $39 \%$ respectively. High or high-normal haematocrit

601 may relate to post-stroke disability, but this is most marked in patients with lacunar infarction.

SUBDURAL EMPYEMA-A SINUSITIS INDUCED DISORDER. CONTINUING DIAGNOSTIC PROBLEMS

J Hodges, P Anslow, G Gillett. Radcliffe Infirmary, Oxford

Subdural empyema is an important but difficult diagnosis. Fourteen cases seen in Oxford since the introduction of the CT scanner are reviewed. Ages ranged from 9 to 68 (mean 26), 10 were male. The clinical presentation was stereotyped and characteristic with persistent drowsiness, focal signs and seizures. The CSF varied from normal to a moderate mixed pleocytosis.

All cases had plain radiological evidence of multiple sinus disease although it was clinically apparent in only a third. This simple investigation was frequently overlooked resulting in considerable diagnostic delay. The CT scan may fail to diagnose the condition and even after repeated scanning only nine patients had clearly identifiable subdural collections.

Two died, one undiagnosed. Thirteen had neurosurgery; craniotomy was the primary procedure in two, five later required craniotomies following burr holes. All had sinus drainage and intensive antibiotic therapy. Streptococcus milleri was isolated in 10. The outcome depends on the promptness of diagnosis and appropriate surgical drainage. The clinical picture with radiological evidence of sinusitis should suggest the diagnosis.

ALTERATIONS IN LYMPHOCYTE SUBSETS DURING THE COURSE OF EXPERIMENTAL ALLERGIC NEURITIS

JV Brosnan, R Fellowes, RI Craggs, RHM King, TJ Bowley, PK Thomas. Royal Free Hospital School of Medicine, London

The autoimmune disease experimental allergic neuritis (EAN) was induced in 
Lewis rats by the intradermal inoculation of an emulsion of bovine dorsal root and Freund's complete adjuvant in the footpads. The proportions of various leucocyte subsets in the blood, spleen and popliteal lymph nodes were assessed at various times between the induction of disease and recovery. The salient abnormality was that the percentage of MRC OX $8+$ cells in the peripheral blood was significantly reduced during the phase of most severe clinical disease, returning to normal with recovery. Adjuvant controls did not show this change. Significant differences were not observed in the other lymphocyte subsets studied (surface Ig+, Ia + , W3.13+, W3.25 + cells) or in the total white cell count in the blood. The popliteal lymph nodes were enlarged both in animals with EAN and in adjuvant controls and both displayed a decrease in the proportion of W3.25 + lymphocytes in comparison with normal unenlarged nodes. No alterations from normal values were detected in the spleen.

The lymphocytes defined by the OX $8+$ antibody are known to contain suppressor cells. It is of interest that various autoimmune diseases in man also show a decrease in suppressor cell populations during acute episodes with recovery during remissions.

NEUROLOGICAL INVOLVEMENT IN X-LINKED HYPOPHOSPHATAEMIC RICKETS

PG Bradbury, DP Brenton, GM Stern. University College Hospital, London

$\mathrm{X}$-linked hypophosphataemic rickets is a familial form of Vitamin D resistant rickets in which gross boney changes may occur. Wasting and weakness of limb muscles is occasionally found and rarely spinal cord compression has been reported. In this study eleven affected patients were reviewed with regard to neurological involvement. Two patients showed severe spinal disease with evidence of spinal cord compression requiring neurosurgical intervention. The characteristic radiological appearances of laminal overgrowth, and spinal ligamentous calcification and thickening are demonstrated. The management of such lesions may be problematic as cord compression may be found at several levels at presentation, and further difficulties develop even after neurosurgical treatment.

EDGE-LIGHT PUPIL CYCLE TIME: A QUANTIFIABLE AUTONOMIC REFLEX CN Martyn. University Department of Neurology, Edinburgh
Persistent regular oscillations of the pupil can be induced if a slit-lamp is used to focus a narrow beam of light at the pupillary margin. The period of these oscillations (pupil cycle time) is simple to measure and proves remarkably constant in normal subjects $(930 \mathrm{~ms} \pm 100$, mean \pm $\mathrm{SD}, \mathbf{n}=32$ ). We have investigated pupil cycle time as a method of identifying and quantifying efferent pupillary defects in patients with disease of the autonomic nervous system. Individual measurements of pupil cycle time in 98 diabetics were found to correlate well with tests of autonomic function based on cardiovascular reflexes. Mean pupil cycle time was prolonged (1309 $\mathrm{ms} \pm 393, \mathrm{n}=53)$ in diabetics with abnormal, compared with those with normal, cardiovascular reflexes (1106 $\mathrm{ms} \pm 192, \mathrm{n}=45, \mathrm{p}<0.001)$. Significant prolongation of pupil cycle time was found in all six of a group of non-diabetic patients with autonomic failure. In none of these cases was the pupillary reaction to light judged abnormal at clinical examination. Measurement of pupil cuycle time is a simple method of detecting abnormality in a part of the autonomic nervous system which previously could only be investigated quantitatively using elaborate apparatus.

TEMPORAL LOBECTOMY FOR EPILEPSY-THE SEIZURE CHARACTERISTICS IN RELATION TO UNDERLYING PATHOLOGY AND OUTCOME JS Duncan, H Sagar, JM Oxbury. Department of Neurology, Radcliffe Infirmary

The seizure histories of 58 patients treated with temporal lobectomy because of drug resistant epilepsy were examined. Seizure characteristics were correlated with the pathology found in the excised temporal lobes and with the outcome after surgery. The most common pathologies were Ammon's horn sclerosis (AHS) (as defined by Adams and Oxbury), patchy hippocampal neurone loss and indolent gliomas. There was a significant association between early childhood convulsions and AHS. There was a suggestion that deja vu phenomena occurred more frequently with patchy hippocampal neurone loss than with AHS. No other associations were found between lateralisation or the pathology found at surgery and 1) the presence of, or type of simple partial seizures, either occurring in isolation or as an aura, or 2) the occurrence of generalised convulsions. The absence of any simple partial seizures was more likely to have been associated with poor outcome and the occurrence of gastric auras was related to a better chance

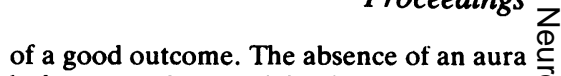
before complex partial seizures carried a 으 higher risk of no improvement after $Z$ surgery. The occurrence of speech auto- $\stackrel{\mathbb{E}}{\subseteq}$ matisms was related to a better chance of a good outcome. Apparently primary generalised convulsions were association with less chance of a good outcome.

\section{CEREBRAL ARTERIOVENOUS}

MALFORMATIONS: NATURAL HISTORY AND EFFECTS OF TREATMENT

P Crawford, C West, MDM Shaw, D Chadwick. Mersey Regional Centre for $\frac{\overrightarrow{7}}{4}$ Medical and Surgical Neurology, Walton Hospital, Liverpool

Several groups have reported the results of $\frac{\rho}{\partial}$ surgery in patients with arteriovenous mal- -2 formations (AVMs). The patients referred $\tilde{\omega}^{N}$ were frequently a selected population; $\frac{1}{0}$ often assessment of outcome after surgical treatment underestimated the morbidity $\frac{\vec{\omega}}{\omega}$ and was rarely compared with conservative $\sigma$ management. The number of untreated patients in these series was usually small, and follow-up limited (Moody 1970; Parkinson 1980; Pellettien 1980; Graft to al 1983). It is essential to define the nature्gl history of AVMs before assessing the results of different management policies. We report the outcome of 343 patients with AVMs presenting to the Merseg Regional Centre for Medical and Surgical Neurology between 1941 and 1984. Tw hundred and forty seven patients presente with haemorrhage, 61 with epilepsy, $\overline{3} \times$ with other neurological symptoms, and inra cr further 12 patients the AVM was a coincidental finding. One hundred and seventeen of these patients were treated surgically. There was a $71 \%$ combined mortality and morbidity rate in those presenting with $\frac{0}{\Phi}$

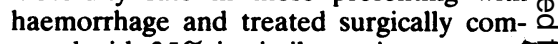
pared with $35 \%$ in similar patients treated $\bar{\sigma}$ conservatively $(p<0.001)$. Operative therapy in this group reduced the risk of subsequent haemorrhage from $36 \%$ to $7 \%$ 贾 ( $p<0.001$ ) but did not significantly affect mortality. There was a $41 \%$ postoperative epilepsy rate $(p<0.001)$, compared with $a$ natural rate of $12 \%$.

MOTOR CONDUCTION VELOCITY IN THE HUMAN SPINAL CORD

Michael Swash, Steven Snooks. The London Hospital and St Mark's Hospital, 을 London

Direct measurement of conduction velocity in the corticospinal tracts in the human $N$ spinal cord should provide a relevant clinical test in patients with spinal cord disease. 
We have achieved this by using transcutaneous spinal stimulation at $\mathrm{C} 6, \mathrm{~L} 1$ and L4 vertebral levels, recording the evoked compound muscle action potential in the pelvic floor musculature (S4 myotome) with surface electrodes in the anal canal or on the tibialis anterior muscle (L5 myotome). This method was developed from the technique of transcutaneous cortical stimulation devised by Merton and colleagues. Supramaximal stimulation was applied through saline soaked pads $5 \mathrm{~cm}$. apart applied with the cathode caudally at the appropriate vertebral level. Single shocks, of 800 to 1,500 volt, decaying with a time constant of 50 us were used. Responses in the pelvic floor muscles (external anal sphincter or puborectalis) or in the tibialis anterior, were reproducible at constant latencies, giving a corticospinal conduction velocity (C6-L1) of $65.9 \pm$ $8.25 \mathrm{~m} / \mathrm{s}$ in 14 normal subjects aged $55 \pm$ 16 years. In these subjects conduction velocity in the cauda equina (L1-L4) was 57.3 $\pm 8.3 \mathrm{~m} / \mathrm{s}$. In a patient with multiple sclerosis and another with radiation myelitis at T6 the corticospinal conduction velocity was 25 and $36 \mathrm{~m} / \mathrm{s}$ respectively. In these two patients conduction velocity in the cauda equina was 76 and $50.8 \mathrm{~m} / \mathrm{s}$ respectively.

\section{NMR IMAGING IN MULTIPLE SCLEROSIS AND CEREBRAL VASCULAR DISEASE: A PRELIMINARY REPORT}

IEC Ormerod, RC Roberts, RW Gilliatt and the Queen Square NMR Group. Institute of Neurology, London

While investigating the specificity of NMR images in multiple sclerosis we have compared the NMR and CT appearances in patients with cerebral vascular disease or MS. We used spin-echo and inversionrểcovery sequences on a Picker 0.25 tesla NMR system. There were 32 patients with clinically definite MS aged 17 to 70 years (mean 41), and 10 patients with cerebral vascular disease aged 23 to 80 (mean 63). In the latter group three patients had presented with epilepsy of late onset; lacunar infarcts had been demonstrated by CT. We ålso examined eight healthy subjects aged 25 to 62 (mean 42). In all the vascular cases there were abnormalities on NMR which included periventricular and separate discrete white matter lesions. In some cases the appearances were indistinguishable from those of MS. The lesions in the patients with cerebral vascular disease tere more extensive on NMR than CT and some lesions which had disappeared from follow up CT scans were obvious on NMR scans three years after presentation. NMR provides a sensitive means of detecting abnormalities in cerebral white matter but with our current sequences the images are not specific.

\section{ACID MALTASE DEFICIENCY IN ADULTS}

P St. J Trend, CM Wiles, GT Spencer, JA Morgan Hughes, BD Lake, AD Patrick. St Thomas's Hospital, Institute of Neurology, Institute of Child Health, London

We have recently encountered five adults with acid maltase deficiency of whom four had presented with acute respiratory failure or chronic nocturnal respiratory insufficiency and had been referred for long term ventilatory support. Despite investigation with muscle biopsy the correct diagnosis had not been made previously in three cases. In retrospect, all patients had developed pelvic girole and truncal weakness in the second or third decade of life followed by evidence of respiratory failure some years later. They were tall, thin individuals with mild neck flexion/extension weakness, severo truncal weakness with paraspinal wasting, mild proximal upper limb weakness but marked weakness of hip abduction and extension. All had paradoxical abdominal wall movements and a fall in vital capacity on lying supine. Muscle biopsy was characteristic in only one case and showed minimal or non-specific features in three. In all five patients glycogen vacuoles were seen in peripheral blood lymphocytes and the diagnosis was confirmed by enzyme assay in muscle, leucocytes or cultured skin fibroblasts. Long term (18 patient-years in 4 patients) domiciliary ventilatory support has proved a highly satisfactory treatment allowing a return to employment. The underlying condition has shown little clinical evidence of progression.

CIRCULATING T CELL SUBSETS IN LAMBERT-EATON MYASTHENIC SYNDROME SA Robb, TJ Bowley, HNA Willcox, J Newsom-Davis. Department of Neurological Science, Royal Free Hospital Medical School, London

LEMS is an autoimmune disease, frequently associated with small cell carcinoma of the lung, in which an IgG autoantibody appears to interfere with acetylcholine release from motor nerve terminals. Because the balance between inducer and suppressor $T$ cell subsets may be disturbed in autoimmune disease, we studied peripheral blood $T$ cell subsets in 15 untreated LEMS patients (10 with small cell carcinoma $(\mathrm{Ca})$, five with no demonstrable tumour (non-Ca)), 10 age matched healthy controls and 10 patients with small cell carcinoma without neurological disease. The proportions of OKT3+ (total) and OKT4+ (helper) $\mathrm{T}$ cells were similar in $\mathrm{Ca}$ LEMS, non-Ca-LEMS and controls. OKT8+ (suppressor/cytotoxic) $T$ cells were significantly decreased in Ca-LEMS compared with non-Ca-LEMS (p < $0.001)$, carcinoma controls $(p<0.01)$ and healthy controls $(p<0.001)$. An increase in OKT8 + $\mathrm{T}$ cells in two of four Ca-LEMS patients studied sequentially was associated with a good response to anti-tumour therapy, but was not seen in the other two patients who died within three months of diagnosis.

The mechanism underlying this relatively selective loss of circulating OKT8+ $T$ cells in Ca-LEMS is unknown, but these changes may help to predict both the presence of carcinoma and the course of the disease.

C9 CONCENTRATION IN CSF FROM PATIENTS WITH DEMYELINATION

DAS Compston, BP Morgan, AK Campbell. University Hospital of Wales, Cardiff

Using an immunoradiometric assay, we have found a reduced concentration of the terminal and lytic component of the complement system-C9-in CSF from 85 patients with demyelination $(0.31 \mu \mathrm{g} / \mathrm{ml})$ compared with 71 individuals with other neurological diseases $(1.53 \mu \mathrm{g} / \mathrm{ml}) ; \mathrm{p}<$ $0.0005)$. Lower $C 9$ concentrations occurred in 13 patients with multiple sclerosis in relapse $(0.18 \mu \mathrm{g} / \mathrm{ml})$ than in 18 cases in remission or 17 with chronic progressive disease $(0.26 \mu \mathrm{g} / \mathrm{ml}$ and $0.35 \mu \mathrm{g} / \mathrm{ml}$, respectively), and in each of these groups compared with controls with acute or chronic diseases $(2.64 \mu \mathrm{g} / \mathrm{ml}$ and 1.37 $\mu \mathrm{g} / \mathrm{ml}$, respectively). Reduced CSF C9 concentrations were also present in 18 patients with acute $(0.31 \mu \mathrm{g} / \mathrm{ml}), 4$ with inactive $(0.37 \mu \mathrm{g} / \mathrm{ml})$ and 15 with chronic progressive $(0.41 \quad \mu \mathrm{g} / \mathrm{ml}) \quad$ isolated demyelinating lesions. $\mathrm{C} 9$ concentration and $\mathrm{C} 9$ index were more discriminating for the diagnosis of multiple sclerosis than IgG concentration or the IgG index. Since C 9 cannot be synthesized in the CNS, the reduced levels imply local $\mathrm{C} 9$ consumption following activation of the complement system by IgG or damaged myelin itself, with formation of membrane attack complexes, thereby amplifying local tissue damage; 
activation of the complement system could also account for the rapid onset and recovery from symptoms which is characteristic of demyelinating disease.

\section{SUSPECTED AND CLINICALLY DEFINITE} MULTIPLE SCLEROSIS: THE RELATIONSHIP BETWEEN CSF IMMUNOGLOBULINS AND CLINICAL COURSE

A Thompson, M Hutchinson, M Mansfield, EA Martin. Department of Neurology, St Vincents/Adelaide Hospitals, Department of Immunology, St James' Hospital, Dublin

Evoked potentials (visual and auditory) and CSF immunoglobulins were examined in 93 patients with clinically definite multiple sclerosis (MS) and 94 patients with possible MS (suspected and progressive possible). Two methods of electrophoresis, polyacrylamide gel electrophoresis (PAGE) and iso electric focusing with immunofixation (IEF) were used and IEF gave a significantly higher frequency of oligoclonal banding in both clinically definite and possible MS. Patients with possible MS and abnormal evoked potentials had a significantly higher IgG index $(1.18 \pm 0.8)$ and a greater frequency of oligoclonal banding $(68 \%)$ than those with normal evoked potentials (IgG index$0.62 \pm 0.32$, oligoclonal banding $-32 \%$, p $<0.001)$. Patients with suspected MS who had CSF oligoclonal banding at presentation had a significantly higher incidence of further neurological lesions at follow up than those who were oligoclonal negative. In clinically definite MS there was no difference in IgG index or oligoclonal banding between those who followed a benign course and those with a more aggressive form of disease. Thus CSF immunoglobulins show close correlation with the number of lesions in patients with possible MS and have a prognostic value in the suspected group. They do not appear to relate to severity or duration of established disease.

SODIUM-POTASSIUM ATPASE ACTIVITY IN THE DORSAL ROOT GANGLIA IN EXPERIMENTAL DIABETES IN RATS

RJ Green, RHM King, PK Thomas, DN Baron. Royal Free Hospital School of Medicine, London

Observations were made on streptozotocin-induced diabetes in Wistar rats. Previous observations (PK Thomas et al., J Neurol Neurosurg Psychiatry, in press) have established that amino acid uptake by dorsal root ganglia is reduced in diabetic animals. Amino acid uptake depends on a sodium gradient maintained by sodium-potassium ATPase. The activity of this enzyme is known to be reduced in the sciatic nerve in experimental diabetes (PK Das et al., Exp Neurol 51:285-288, 1976). In the present experiments activity was therefore estimated in dorsal root ganglia. A $50 \%$ reduction in ouabainsensitive sodium-potassium ATPase activity was found in excised diabetic ganglia in comparison with those from age-matched control animals. This finding may be relevant to the causation of diabetic neuropathy by leading to an interference with perikaryal protein synthesis.

OBSERVATIONS ON POSTPRANDIAL

HYPOTENSION AND ITS CAUSATION IN

AUTONOMIC FAILURE

DF da Costa, R Bannister, CJ Mathias. St Mary's Hospital, London, W2

The ingestion of food accentuates symptoms of orthostatic hypotension in many patients with idiopathic chronic autonomic failure (AF). We therefore studied the magnitude and mechanisms of postprandial hypotension (PPH) in a series of patients with AF. Cardiovascular, hormonal and biochemical measurements were made in six patients with AF before and after a standard breakfast in the horizontal position. Blood pressure (BP) fell markedly, within 15 minutes, from $145 \pm 9 / 80 \pm 5$ $\mathrm{mmHg}$ to the lowest level of $82 \pm 3 / 47 \pm 4$ $\mathrm{mmHg}$ after 60 mins and even after three hours BP was still well below basal levels ( $p$ $<0.05)$. No BP changes occurred in six normal subjects. In AF, plasma noradrenaline (NA) and acrenaline (A) levels were low and did not change postprandially, unlike in the normal subjects where plasma NA rose significantly. The haematocric (hct) and plasma osmolality (osm) remained unchanged excluding a major shift of fluid from the intravascular compartment. Food ingestion therefore causes a substantial fall in blood pressure which is not counteracted by an increase in sympathetic nervous activity. To ascertain which component of the meal was responsible for these effects we studied the cardiovascular and biochemical responses to carbohydrate ingestion. Exogenous insulin lowers blood pressure in autonomic impairment due to either diabetes mellitus (Page and Watkins 1976, Diabetes 25, 90) and tetraplegia (Mathias et al 1979, Paraplegia, 17,319). We therefore studied the responses to an oral glucose solution (G, $1 \mathrm{~g} / \mathrm{kg}$ in $300 \mathrm{ml} \mathrm{H} \mathrm{O}$ ) and an iso-osmotic xylose solution $(\mathrm{X})$, which does not release insulin, in six patients with AF. After $G_{\frac{C}{C}}^{\mathbb{D}}$ BP was lowered within 15 minutes with maximum effect after one hour, 165 $8 / 87 \pm 4$ to $109 \pm 8 / 63 \pm 4,(p<0.01) \Phi$ The BP remained lower than the basaf level when the study terminated after tw hours. The results were therefore similar in magnitude and duration to those caused by a meal. Xylose caused a much smaller an $e^{4}$ transient fall in BP. There were no change $\$$ in hct, plasma osm and electrolytes. Or glucose did not change BP in normal sub jects.

Our studies indicate that food ingestio $\overrightarrow{\overrightarrow{\mathrm{s}}}$ causes a marked fall in BP in patients withit AF. It is likely that the carbohydrate com ponent of the meal, possibly by its insulir releasing effects, plays a major role in the causation of postprandial hypotension.

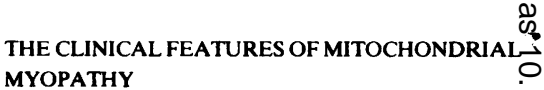

RKH Petty, AE Harding, JA Morgan Hughes. Institute of Neurology an National Hospital for Nervous Diseases Queen Square, London

Sixty-one patients with histologicalfy defined mitochondrial myopathy have be्हn investigated at the National Hospital ot the period $1969-84$, and more than 4005 these have been reviewed in the last year? The mean age of onset of symptoms was $22-$ years, ranging from birth to 69 years. Sixty percent presented with ocular symptongs of which ptosis was the most frequent Twenty-five per cent of patients had simiteo larly affected relatives. Three groups:of cases could be identified clinically: a combination of progressive external ophthalmoplegia with fatigable weakness of the limbs $(54 \%)$, fatigable limb weakness alone $(18 \%)$, and those with clinical features (such as ataxia, dementia, pyramidab signs, involuntary movements and seizures predominantly or exclusively confined to 3 the central nervous system $(28 \%)$. There was some overlap between these groups Pigmentary retinopathy, present in $30 \%$ of cases, occurred in all three. Mitichondria metabolism was studied in 23 cases; no typ $=-\sigma^{4}$ ical clinical picture emerged for any of the identifiable biochemical defects. At a mear? disease duration of 20 years, ranging from 2 to 50 years, $15 \%$ of patients were severely disabled but $65 \%$ were still able to work.

IS TRANSIENT GLOBAL AMNESIA A FORM OF CEREBRAL ISCHAEMIC ATTACK?

J Hodges, CP Warlow. Department o Neurology, Radcliffe Infirmary, Oxford 
The aetiology of transient global amnesia (TGA) is obscure and the natural history disputed. Many feel they represent transient cerebral ischaemic attacks (TIA). In an attempt to answer these questions we performed a case-control follow-up study. Fifty patients with TGA fulfilling strict diagnostic criteria (26 females and 24 males, ages $40-84$, mean 63.5 ) and 100 age and sex mached TIA controls; (64 carotid, 29 vertebrobasilar and 29 amaurosis) were compared. Mean follow-up periods were 31.6 and 28.6 months respectively. In the TGA group $10 \%$ had prior symptoms of cerebral ischaemia, $14 \%$ treated hypertension, $6 \%$ ischaemic heart disease and $8 \%$ carotid bruits. Of TIA patients $42 \%$ had hypertension, $23 \%$ ischaemic heart disease and $31 \%$ carotid bruits. TGA recurred in $30 \%$ and $6 \%$ became manifestly epileptic. Cerebral ischaemic events (stroke or TIA) occurred in $4 \%$ of TGA patients ( 1.5 per 100 patient years) and $29 \%$ of TIA patients (12.2 per 100 patient years). The $95 \%$ confidence intervals for these two rates were $0-4.9$ and 5.8-18.6 events per patient year respectively. In the TIA group $4 \%$ had myocardial infarcts and $6 \%$ vascular deaths whereas neither event occurred in TGA patients.

TGA appears to be a syndrome of mixed aetiology, only a minority have evidence of vascular disease and these should be identifiable. The prognosis for the remainder is excellent.

ÓCULAR MOTOR AND HAND TRACKING IN PARKINSONS DISEASE AND THEIR RESPONSE TO THERAPY

JM Gibson, RM Pimlott, C Kennard. The London Hospital, London

Fifteen previously untreated Parkinsonian patients were clinically rated and had their horizontal saccadic and smooth pursuit eye movements assessed using infra-red oculography. A tracking task was used to study hand performance. Computer Enalysis generated stimulus-response statistics which were compared with normal aged-matched controls. Individual sac- cades were found to have normal peak velocities, but were inaccurate and had mildly prolonged latencies which showed a greater scatter than the controls. Smooth pursuit gain of the eye was significantly reduced. In the hands, although there was some impairment in all the rapid step movements, a decrease in the smooth pursuit gain was the most marked finding. All patients were followed on treatment. Clinically improved patients showed improvements in saccadic accuracy and smooth pursuit gain of the eye. Analysis of hand movements revealed a modest improvement in latency, and considerable elevations in smooth pursuit gain. A separate study of five patients, with marked on/off fluctuations revealed similar changes in performance during the on/off periods. It is concluded that certain aspects of ocular motor function in Parkinson's disease are abnormal, but can be improved with treatment. Quantitative study of smooth pursuit of the eye and limb is a valuable method for the objective measurement of akinesia.

PATHOPHYSIOLOGY OF CRANIAL DYSTONIA A Berardelli, JC Rothwell, BL Day, CD Marsden. Institute of Psychiatry, London

The physiology of reflexes mediated by the fifth and seventh cranial nerves has been studied in 16 patients with blepharospasm and oromandibular dystonia. The EMG activity associated with the spasms in the orbicularis oculi and jaw muscles was similar to that described in other muscles in patients with torsion dystonia. The latency of the R1 and R2 components of the blink and of the corneal reflexes was normal, but the duration of the R2 and of the corneal reflex was longer than in normal subjects. In some patients the $R 1$ component also was present on the side contralateral to the stimulus. The excitability cycle of the R2 component of the blink reflex was enhanced. There were no EEG potentials preceding involuntary spasms, although a Bereitschafts potential was seen beginning some $500 \mathrm{~ms}$ prior to voluntary blinks in the same individuals. The exteroceptive suppression in the masseter and in the orbicularis oculi muscles was absent in $40-50 \%$ of the patients. The jaw jerk was present in all the patients with normal latency.

These results indicate that the reflex arcs of the facial reflexes are normal. However, we suggest that an excitatory drive from the basal ganglia is exerted on the interneurones which mediate the facial reflexes in the brain stem.

\section{TRACE ELEMENT STUDIES IN MOTOR} NEURONE DISEASE

JD Mitchell, BW East, IA Harris, B Pentland. Department of Medical Neurology, Northern General Hospital, and Scottish Universities Research and Reactor Centre, Glasgow

It seems increasingly likely that MND is the result of a metabolic abnormality selectively affecting cells of the motor system. A defect of enzymatic DNA repair mechanisms has recently been postulated. In view of this we have studied trace elements in CSF, spinal cord, liver and bone in a control population and a series of MND patients. The CSF cobalt content was reduced in the patient group $(p=0.0015)$. In the spinal cord the levels of selenium ( $p$ $=0.0005)$, manganese $(p=0.000082)$ and cobalt $(p=0.0072)$ were increased in the patient group. The hepatic selenium content was also increased in the patient group $(p=0.014)$. These findings are clearly tantalising in our attempts to understand this mysterious disease. One must guard against over-interpreting them. Although the biological role of cobalt as a constituent of cobalamin is well recognised, its function in other forms is controversial in higher animals. In bacteria, however, elemental cobalt seems to be important in nucleic acid metabolism. Manganese and selenium are involved in the degradation of free radicals. The results support attempts to find an abnormality of nucleic acid metabolism, enzymatic or otherwise in MND and also work examining the role of free radicals in this condition. 